\title{
Neutrophil-lymphocyte ratio acts as a novel diagnostic biomarker for kidney stone prevalence and number of stones passed
}

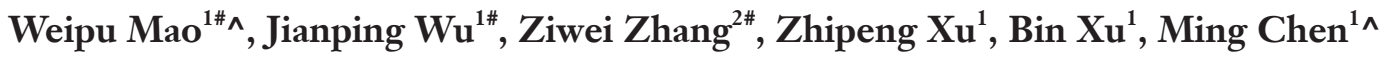 \\ ${ }^{1}$ Department of Urology, Affiliated Zhongda Hospital of Southeast University, Nanjing, China; ${ }^{2}$ Department of Urology, Changhai Hospital, Second \\ Military Medical University, Shanghai, China \\ Contributions: (I) Conception and design: W Mao, B Xu, M Chen; (II) Administrative support: Z Zhang, Z Xu; (III) Provision of study materials \\ or patients: W Mao, J Wu; (IV) Collection and assembly of data: W Mao, Z Zhang; (V) Data analysis and interpretation: W Mao, J Wu; (VI) \\ Manuscript writing: All authors; (VII) Final approval of manuscript: All authors. \\ \#These authors contributed equally to this work. \\ Correspondence to: Ming Chen, PhD; Bin Xu, PhD. Department of Urology, Affiliated Zhongda Hospital of Southeast University, No. 87 Dingjiaqiao, \\ Hunan Road, Gulou District, Nanjing 210009, China. Email: mingchenseu@126.com; njxb1982@126.com.
}

Background: This study evaluated the relationship between inflammatory biomarkers and the prevalence of kidney stones and number of stones passed.

Methods: We conducted a cross-sectional study of adult participants ( $\geq 20$ years) in the National Health and Nutrition Examination Survey (NHANES) from 2007 to 2014. We calculated key inflammatory biomarkers, such as the systemic immune-inflammation index (SII), neutrophil-lymphocyte ratio (NLR), plateletlymphocyte ratio (PLR) and monocyte-lymphocyte ratio (MLR). Multivariate logistic regression analysis was used to determine the effect of inflammatory biomarkers on the prevalence of kidney stones and number of stones passed.

Results: A total of 21,106 participants were included in the final study, and 1,864 patients reported a history of kidney stones (including 292 who had passed no stones, 1,462 who had passed stones 1-5 times, and 110 who had passed stones $>5$ times). The chi-square test showed that the NLR, MLR and SII were closely related to the occurrence of kidney stones and the number of stones passed. Multivariate logistic regression analysis showed that a high NLR (>1.72) was associated with an increased prevalence of kidney stones and number of stones passed ( $\mathrm{OR}=1.18,95 \% \mathrm{CI}: 1.03-1.36, \mathrm{P}=0.019)$.

Conclusions: A convenient biomarker, the NLR can be used as a good predictor for the diagnosis of kidney stones and number of stones passed; these findings are worthy of further research and application in clinical practice.

Keywords: Kidney stone; neutrophil-lymphocyte ratio; prevalence; stone pass number; National Health and Nutrition Examination Survey

Submitted Apr 26, 2020. Accepted for publication Sep 25, 2020.

doi: 10.21037/tau-20-890

View this article at: http://dx.doi.org/10.21037/tau-20-890

\section{Introduction}

One of the most common urinary diseases is nephrolithiasis, which refers to stones that occur in the renal calyx, renal pelvis and the junction of the renal pelvis and ureter, and its incidence is increasing worldwide (1). Nephrolithiasis occurs when the balance between solubility and precipitation of stone-forming salts in the urinary tract and kidney is disrupted (2). Between 2013 and 2014, the adjusted

\footnotetext{
^ ORCID: Weipu Mao, 0000-0002-2455-4396; Ming Chen, 0000-0002-3572-6886.
} 
prevalence of kidney stones in Chinese adults was 5.8\% (3). Nephrolithiasis typically involves repeated occurrences, which indicates a higher recurrence rate and worse clinical prognosis (4).

There are few studies on the role of immune responses and inflammatory cells in the formation of kidney stones. Some studies have shown that the retention of crystals is mediated by excessive production of reactive oxygen species and subsequent oxidative stress and inflammation (5-7). The levels of neutrophils, platelets, lymphocytes and acute phase proteins change during this inflammatory response. There are many inflammatory indicators that can be combined to function as biomarkers, including the systemic immune-inflammation index (SII), neutrophillymphocyte ratio (NLR), platelet-lymphocyte ratio (PLR) and monocyte-lymphocyte ratio (MLR). Considering the growing evidence of the relationship between kidney stones and inflammatory diseases, we examined whether the levels of key inflammatory biomarkers mentioned above are associated with the prevalence of kidney stones and the number of stones passed

We present the following article in accordance with the STROBE reporting checklist (available at http://dx.doi. org/10.21037/tau-20-890).

\section{Methods}

\section{Study population}

The National Health and Nutrition Examination Survey (NHANES) uses a complex, probability-based sampling design to collect information from different populations through standardized interviews, physical examinations, and laboratory tests to assess the health and nutritional status of noninstitutionalized civilians in the United States. Since 1999, the data have been released every two years for research use.

In this study, we used public data from four NHANES cycles (2007-2008, 2009-2010, 2011-2011 and 20132014). More information about the data are available on the NHANES website (www.cdc.gov/nchs/nhanes/). Between 2007 and 2014, there were 40,617 participants in NHANES. We first excluded 30 participants who reported a history of hematological tumors $(n=7)$ or leukemia $(n=23)$. The subsequent exclusion criteria were as follows: (I) incomplete stone investigation ( $\mathrm{n}=17,201)$; (II) unknown platelet count ( $\mathrm{n}=1,926)$; (III) unknown lymphocyte count $(\mathrm{n}=43)$; (IV) unknown/abnormal neutrophil count $(\mathrm{n}=2)$;
(V) unknown body mass index (BMI) $(\mathrm{n}=269)$; and (VI) unknown number of kidney stone occurrences $(n=40)$. After excluding these factors, 21,106 participants were included in the final study.

The study was conducted in accordance with the Declaration of Helsinki (as revised in 2013). This study used previously collected deidentified data, which was deemed exempt from review by the Ethics Committee of the Affiliated Zhongda Hospital of Southeast University.

\section{Study variables and outcome}

The SII, NLR, PLR and MLR are the main predictors of outcomes measured in this study. The SII is calculated as the platelet count $\times$ neutrophil count/lymphocyte count; the NLR is the neutrophil count/lymphocyte count; the PLR is the platelet count/lymphocyte count; and the MLR is the monocyte count/lymphocyte count. The lymphocyte count, platelet count and monocyte count can be obtained directly from laboratory data files. The neutrophil count is calculated from the white blood cell count and neutrophil percentage. Other covariates included sex (male or female), age (20-39 years, 40-59 years and 60+ years), race (nonHispanic white, non-Hispanic Black, Mexican American, other Hispanic and other), marital status (married, unmarried and other), participation in participation in vigorous recreational activities (yes or no), participation in moderately or vigorous recreational activities (yes or no), education level (less than high school, high school or equivalent, college or above and other), and BMI $(<25.0$, $25.0-29.9$ and $\geq 30.0 \mathrm{~kg} / \mathrm{m}^{2}$ ). Hypertension and diabetes are defined as self-reported physician-diagnosed hypertension or diabetes.

The end points of the study were the history of kidney stones and the occurrence of kidney stone episodes. The end points were extracted from the questionnaire data file. Participants who answered "No" to the question "Have you ever had kidney stones?" were considered to have no history of kidney stones. For participants who answered "Yes", we also documented their answer to the "How many times have you passed a kidney stone?" question. For those who answered " 0 ", we considered them as not having passed stones, and for those who answered " $1-80$ ", we considered them as having passed stones.

\section{Statistical analysis}

The participants were divided into four groups: the no 


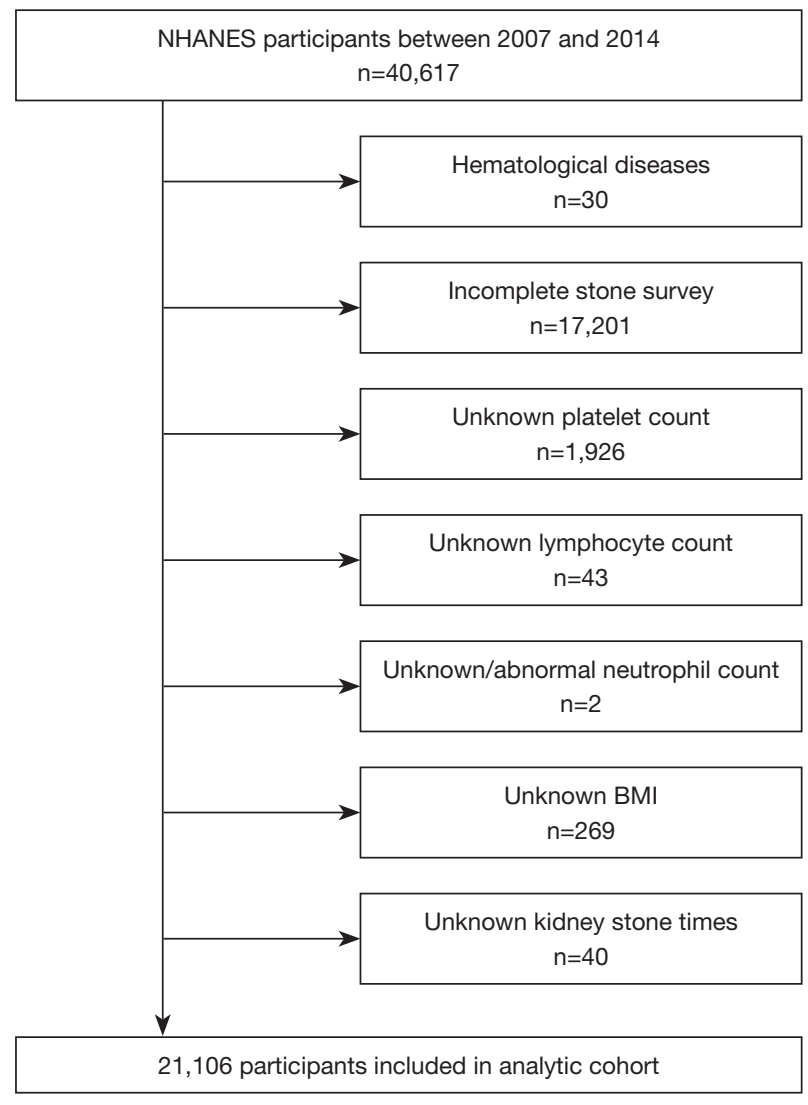

Figure 1 Schematic overview for patient identification.

stone group, the no-stones-passed group, the stones-passed 1-5 times group and the stones-passed $>5$ times group. We used MedCalc software (version 15.2.0) to determine the cutoff values for the SII, NLR, PLR, and MLR. The mean \pm standard deviation is used to describe the distribution of continuous variables, and the proportion is used to describe the distribution of categorical variables. Chi-square analysis was used to evaluate the clinical characteristics of all participants. A multivariate logistic regression model was used to evaluate the adjusted odds ratio (OR) and $95 \%$ confidence interval (CI) of factors related to kidney stones and the number of kidney stones passed. All statistical analyses were performed using SPSS (version 24.0), and graphs were generated by GraphPad (version 8.0.2). $\mathrm{P}<0.05$ (two-tailed) was considered to indicate statistical significance.

\section{Results}

Based on the screening criteria in Figure 1, we included
21,106 qualified participants from the NHANES database from 2007 to 2014. Table 1 shows the clinical characteristics of all participants. Among them, 19,242 were in the no stone group, 292 in the no-stones-passed group, 1,462 in the stones passed 1-5 times group and 110 in the stones passed $>5$ times group. The chi-square test showed significant differences among several variables, including sex $(\mathrm{P}<0.001)$, age $(\mathrm{P}<0.001)$, race $(\mathrm{P}<0.001)$, marital status $(\mathrm{P}<0.001)$, participation in moderately or vigorous recreational activities $(\mathrm{P}<0.001)$, hypertension $(\mathrm{P}<0.001)$, diabetes $(\mathrm{P}<0.001)$, BMI $(\mathrm{P}=0.004)$, the SII $(\mathrm{P}=0.001)$, the NLR $(\mathrm{P}<0.001)$ and the MLR $(\mathrm{P}<0.001)$. We found that the proportion of males, non-Hispanic whites, no participation in activities, married, college or above, hypertension, diabetes/borderline, obese, SII $>44.37$, NLR $>1.72$ and MLR $>0.29$ in the group with stones passed $>5$ times was significantly higher than that in the other three groups.

We focused on investigating the expression of four inflammatory biomarkers (the SII, NLR, PLR and MLR) in the four groups. We found that the levels of the SII $(534.26 \pm 336.33,545.91 \pm 370.32,557.27 \pm 330.87$, and $613.39 \pm 405.50)$ and NLR $(2.18 \pm 1.94,2.30 \pm 1.19,2.35 \pm 1.30$, and $2.52 \pm 1.31$ ) increased progressively (Figure 2 ) in the no stone group, no stones passed group, stones passed 1-5 times group and stones passed $>5$ times group, and the proportion of participants with the $\mathrm{SII}>44.37, \mathrm{NLR}>1.72$ and MLR $>0.29$ showed an increase in each subsequent group (Figure 3).

To further determine the risk factors related to the prevalence of kidney stones and the number of stones passed, we subsequently conducted a multivariate logistic regression analysis (Table 2). The results showed that the prevalence of kidney stones and the number of stones passed were related to sex, age, race, participation in moderately or vigorous recreational activities, BMI and the NLR. We found that female participants had a lower risk of kidney stones ( $v s$. male participants $\mathrm{OR}=0.73,95 \%$ CI: 0.66-0.81, $\mathrm{P}<0.001)$. Patients aged $40-59$ years (vs. 20 39 years $\mathrm{OR}=1.21,95 \%$ CI: $1.08-1.36, \mathrm{P}=0.001), 60+$ years (vs. $20-39$ years $\mathrm{OR}=1.83,95 \%$ CI: $1.58-2.13, \mathrm{P}<0.001$ ), who did not participate in activities ( $v s$. participation in activities $\mathrm{OR}=1.20,95 \% \mathrm{CI}$ : $1.03-1.40, \mathrm{P}=0.017$ ), who did not participate in moderately vigorous recreational activities (vs. participation in moderately vigorous recreational activities $\mathrm{OR}=1.13,95 \% \mathrm{CI}: 1.02-1.26, \mathrm{P}=0.022)$, who were overweight ( $v s$. normal OR $=1.15,95 \%$ CI: $1.03-1.29$, $\mathrm{P}=0.015$ ) and who had obesity ( $v s$. normal $\mathrm{OR}=1.50,95 \%$ 
Table 1 Baseline characteristics of 21106 NHANES participants between 2007 and 2014

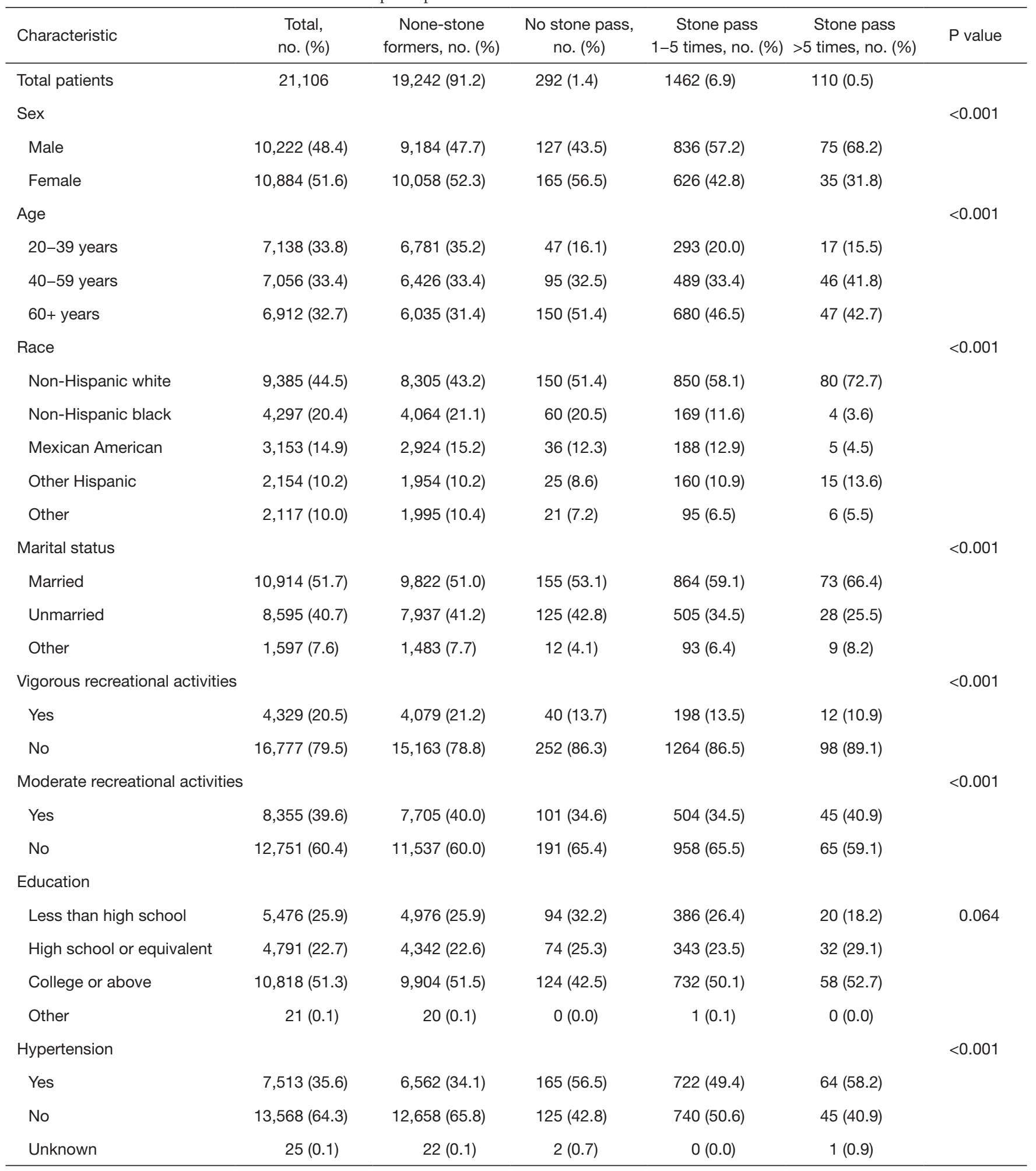

Table 1 (continued) 
Table 1 (continued)

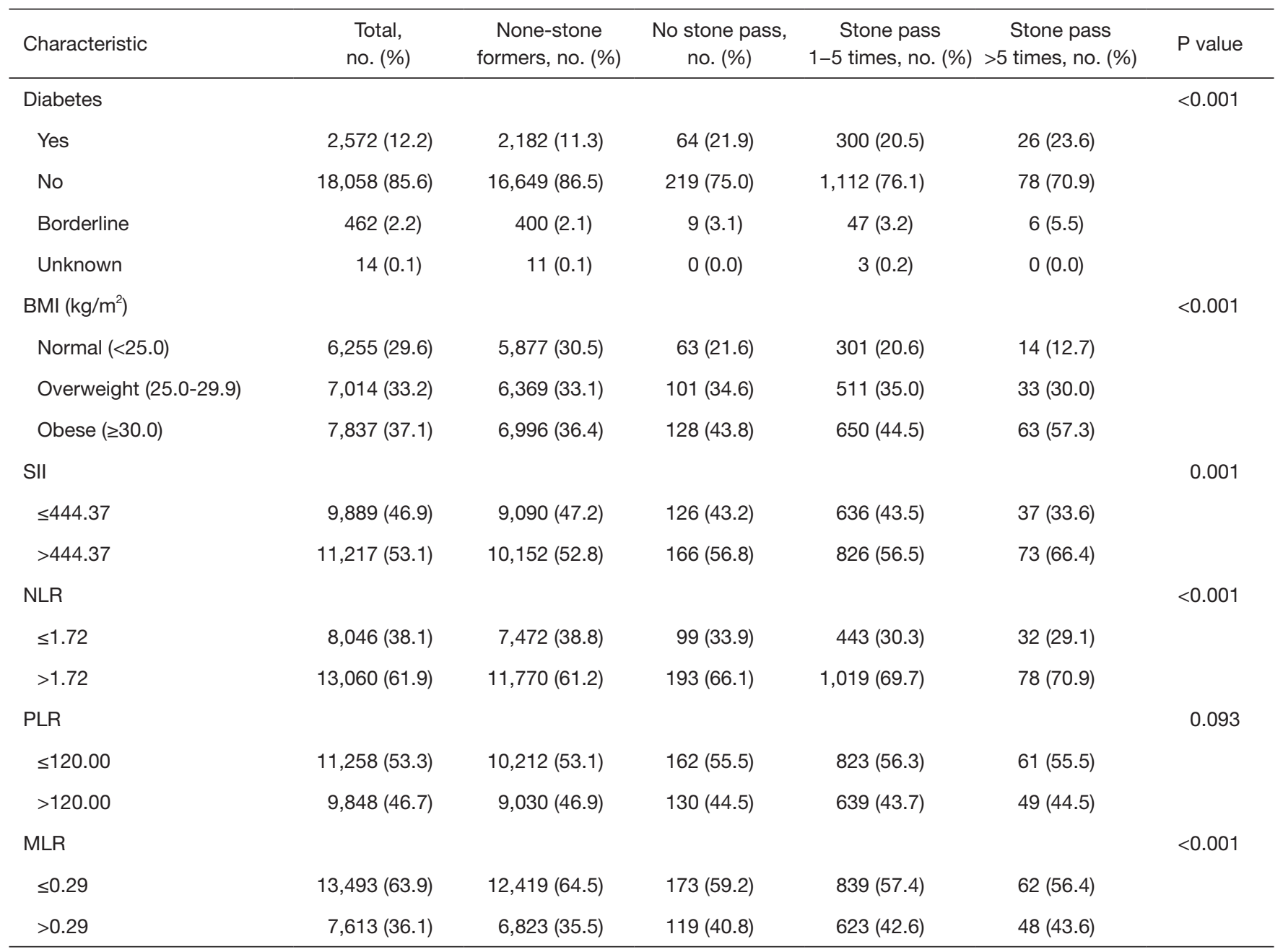

BMI, body mass index; SII, systemic immune-inflammation index; NLR, neutrophil-lymphocyte ratio; PLR, platelet-lymphocyte ratio; MLR, monocyte-lymphocyte ratio.

CI: $1.31-1.71, \mathrm{P}<0.001)$ had a higher risk of kidney stones. In addition, of the four inflammatory biomarkers, only the NLR was associated with the prevalence of kidney stones and the number of stones passed (NLR $>1.72 v s$. NLR $\leq 1.72$ OR $=1.18,95 \%$ CI: $1.03-1.36, \mathrm{P}=0.019$ ).

\section{Discussion}

In this retrospective study, we used the NHANES database, which can be assumed to represent the population of the United States. Our results showed for the first time that the SII, NLR and MLR increased, and the proportion of the SII $>444.37$ and MLR $>0.29$ increased as the incidence of kidney stones increased from the no-stone group, nostones-passed group, stones passed 1-5 times group and stones passed $>5$ times group. There was a significant correlation between higher SII, NLR and MLR values and the self-reported prevalence of kidney stones and the number of stones passed. After adjusting for other confounding factors, only the NLR was associated with the prevalence of kidney stones and the number of stones passed. This indicates that the upregulated NLR can be regarded as a powerful indicator for the diagnosis of kidney stone prevalence and number of stones passed.

Kidney stones are a common and frequently occurring condition that seriously affects human health, and as the 
A



C

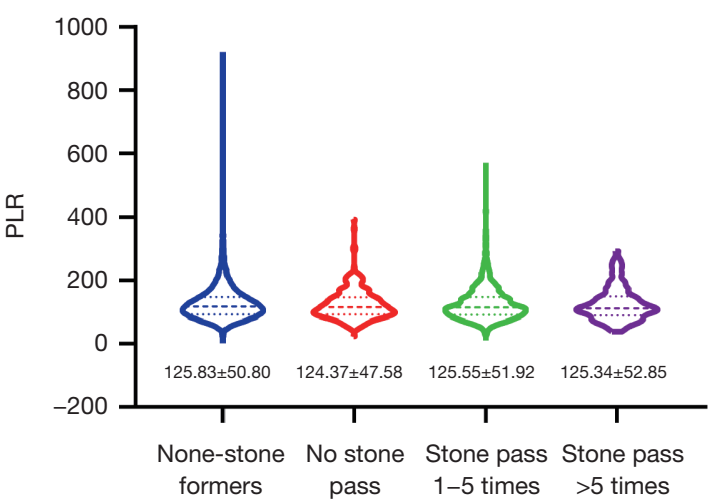

B

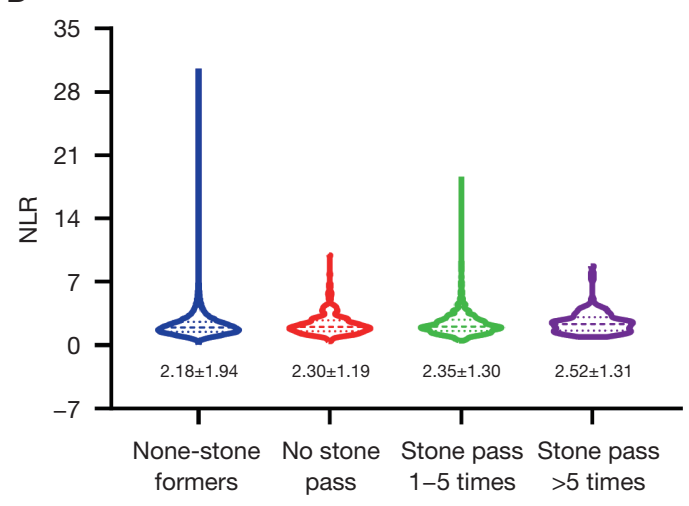

$\mathrm{D}$

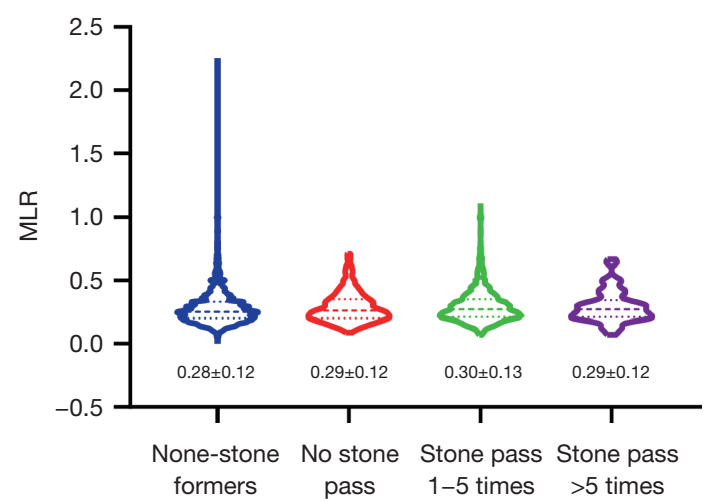

Figure 2 The values of SII, NLR, PLR and MLR for different groups. (A) SII; (B) NLR; (C) PLR; (D) MLR. SII, systemic immuneinflammation index; NLR, neutrophil-lymphocyte ratio; PLR, platelet-lymphocyte ratio; MLR, monocyte-lymphocyte ratio.

prevalence of stones increases, the cost of medical care related to stones significantly increases as well (8). Among kidney stones, calcium oxalate stones are the most common, accounting for approximately $80 \%$ (9). The recurrence rate of kidney stones is very high, $50 \%$ in $5-10$ years, and patients with recurrent stones are more likely to have more recurrences (10). The specific pathogenesis of kidney stones is still unclear. In recent years, the role of oxidative stress and the inflammatory response in the occurrence and development of kidney stones has provoked widespread concern among scholars. An increasing number of studies have confirmed that oxidative stress and inflammatory responses are closely related to the formation of kidney stones $(6,11,12)$.

Oxidative stress and inflammatory responses play an important role in a variety of crystal-related diseases, such as gout (uric acid crystals), silicosis (silica crystals), and atherosclerosis (cholesterol crystals) (13-15). Kidney stones are also a crystal-related disease, and oxalate crystals and other components in urine can lead to oxidative stress and inflammatory responses in tissue and cells, resulting in the release of inflammatory cells and inflammatory factors (16).

During inflammation, the levels of neutrophils, lymphocytes and C-reactive protein (CRP) change. Neutrophils can release inflammatory factors (such as IL-8, IL-6, and neutrophil elastase), and lymphocytes can secrete cytokines (e.g., interferon- $\gamma$ and tumor necrosis factor- $\alpha$ ) (17). As a simple peripheral blood indicator that can better reflect the level of systemic inflammation compared with lymphocytes, an increase in the NLR indicates an increase in neutrophils, which promote inflammation (18). As a biomarker of acute and chronic phase inflammation 
A

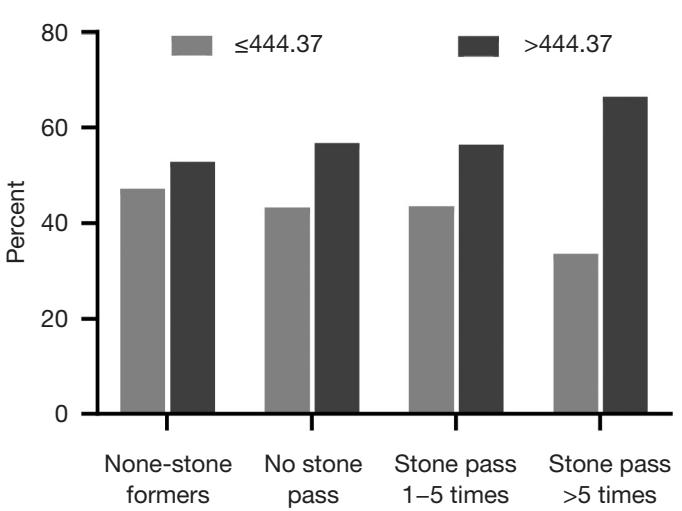

C



B $\quad$ NLR
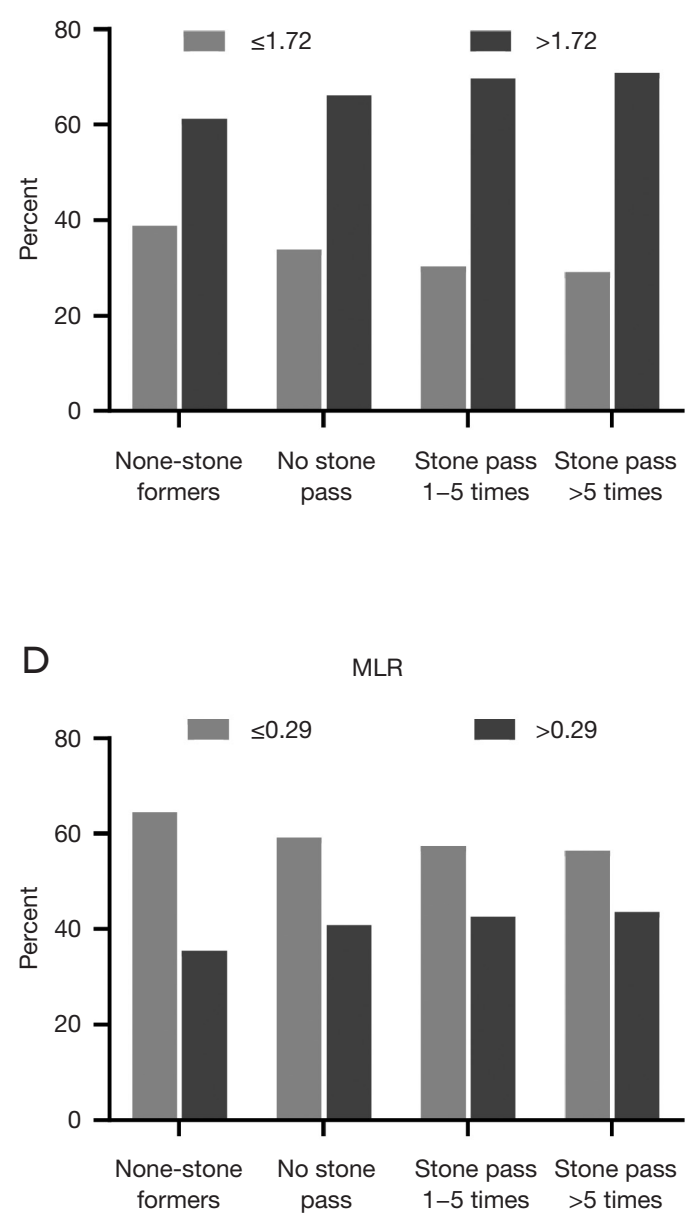

Figure 3 The proportion of SII, NLR, PLR and MLR in different groups. (A) SII; (B) NLR; (C) PLR; (D) MLR. SII, systemic immuneinflammation index; NLR, neutrophil-lymphocyte ratio; PLR, platelet-lymphocyte ratio; MLR, monocyte-lymphocyte ratio.

$(19,20)$, CRP has been proven to be associated with kidney stones. Shoag et al. (21) found a significant correlation between serum CRP levels and self-reported kidney stones in younger individuals.

Because routine blood examination is economical, easy to perform and encompasses a large number of useful parameters, it should be fully applied in the diagnosis and management of kidney stones, which is worthy of further discussion.

Our research also has certain limitations. First, our study is based on the NHANES database, which is a crosssectional study; further research requires a prospective study. Second, there is no information in the NHANES database about the type of kidney stones. Moreover, NHANES survey results are based on participants' selfreported data and require the use of a diagnostic test; further, the complete kidney stone history is not available.

\section{Conclusions}

Overall, this study found that a higher NLR was closely associated with self-reported kidney stones and the number of stones passed. Because the NLR is a convenient and economical biomarker derived from a routine blood examination, it may play an important role in the screening and prevention of kidney stones in future clinical practice. 
Table 2 Multivariate logistical regression analysis of risk factors

\begin{tabular}{|c|c|c|}
\hline Characteristic & Adjusted OR (95\% Cl) & $P$ value \\
\hline \multicolumn{3}{|l|}{ Sex } \\
\hline Male & Reference & \\
\hline Female & $0.73(0.66-0.81)$ & $<0.001$ \\
\hline \multicolumn{3}{|l|}{ Age } \\
\hline 20-39 years & Reference & \\
\hline $40-59$ years & $1.21(1.08-1.36)$ & 0.001 \\
\hline $60+$ years & $1.83(1.58-2.13)$ & $<0.001$ \\
\hline \multicolumn{3}{|l|}{ Race } \\
\hline Non-Hispanic white & Reference & \\
\hline Non-Hispanic black & $0.58(0.47-0.70)$ & $<0.001$ \\
\hline Mexican American & $1.35(1.07-1.70)$ & 0.012 \\
\hline Other Hispanic & $0.94(0.74-1.17)$ & 0.585 \\
\hline Other & $0.70(0.55-0.89)$ & 0.004 \\
\hline \multicolumn{3}{|l|}{ Marital status } \\
\hline Married & Reference & \\
\hline Unmarried & - & 0.776 \\
\hline Other & - & 0.114 \\
\hline \multicolumn{3}{|l|}{ Vigorous recreational activities } \\
\hline Yes & Reference & \\
\hline No & $1.20(1.03-1.40)$ & 0.017 \\
\hline \multicolumn{3}{|c|}{ Moderate recreational activities } \\
\hline Yes & Reference & \\
\hline No & $1.13(1.02-1.26)$ & 0.022 \\
\hline \multicolumn{3}{|l|}{ Education } \\
\hline Less than high school & Reference & \\
\hline High school or equivalent & - & 0.652 \\
\hline College or above & - & 0.613 \\
\hline Other & - & 0.617 \\
\hline \multicolumn{3}{|l|}{ Hypertension } \\
\hline Yes & Reference & \\
\hline No & - & 0.856 \\
\hline Unknown & - & 0.472 \\
\hline \multicolumn{3}{|l|}{ Diabetes } \\
\hline Yes & Reference & \\
\hline No & - & 0.265 \\
\hline Borderline & - & 0.086 \\
\hline Unknown & - & 0.207 \\
\hline
\end{tabular}

Table 2 (continued)
Table 2 (continued)

\begin{tabular}{|c|c|c|}
\hline Characteristic & Adjusted OR (95\% Cl) & $P$ value \\
\hline \multicolumn{3}{|l|}{$\mathrm{BMI}\left(\mathrm{kg} / \mathrm{m}^{2}\right)$} \\
\hline Normal $(<25.0)$ & Reference & \\
\hline Overweight (25.0-29.9) & $1.15(1.03-1.29)$ & 0.015 \\
\hline Obese $(\geq 30.0)$ & $1.50(1.31-1.71)$ & $<0.001$ \\
\hline \multicolumn{3}{|l|}{ SII } \\
\hline$\leq 444.37$ & Reference & \\
\hline$>444.37$ & - & 0.398 \\
\hline \multicolumn{3}{|l|}{ NLR } \\
\hline$\leq 1.72$ & Reference & \\
\hline$>1.72$ & $1.18(1.03-1.36)$ & 0.019 \\
\hline \multicolumn{3}{|l|}{ PLR } \\
\hline$\leq 120.00$ & Reference & \\
\hline$>120.00$ & - & 0.545 \\
\hline \multicolumn{3}{|l|}{ MLR } \\
\hline$\leq 0.29$ & Reference & \\
\hline$>0.29$ & - & 0.928 \\
\hline
\end{tabular}

BMI, body mass index; SII, systemic immune-inflammation index; NLR, neutrophil-lymphocyte ratio; PLR, platelet-lymphocyte ratio; MLR, monocyte-lymphocyte ratio.

\section{Acknowledgments}

The authors are grateful for the invaluable support and useful discussions with other members of the urological department.

Funding: This work was supported by the National Natural Science Foundation of China (No. 81572517 and 81872089), Natural Science Foundation of Jiangsu Province (BK20161434), Jiangsu Provincial Medical Innovation Team (CXTDA2017025), and Jiangsu Provincial Medical Talent (ZDRCA2016080).

\section{Footnote}

Reporting Checklist: The authors have completed the STROBE reporting checklist. Available at http://dx.doi. org/10.21037/tau-20-890

Conflicts of Interest: All authors have completed the ICMJE uniform disclosure form (available at http://dx.doi. 
org/10.21037/tau-20-890). The authors have no conflicts of interest to declare.

Ethical Statement: The authors are accountable for all aspects of the work in ensuring that questions related to the accuracy or integrity of any part of the work are appropriately investigated and resolved. The study was conducted in accordance with the Declaration of Helsinki (as revised in 2013). This study used previously collected deidentified data, which was deemed exempt from review by the Ethics Committee of the Affiliated Zhongda Hospital of Southeast University.

Open Access Statement: This is an Open Access article distributed in accordance with the Creative Commons Attribution-NonCommercial-NoDerivs 4.0 International License (CC BY-NC-ND 4.0), which permits the noncommercial replication and distribution of the article with the strict proviso that no changes or edits are made and the original work is properly cited (including links to both the formal publication through the relevant DOI and the license). See: https://creativecommons.org/licenses/by-nc-nd/4.0/.

\section{References}

1. Sorokin I, Mamoulakis C, Miyazawa K, et al. Epidemiology of stone disease across the world. World J Urol 2017;35:1301-20.

2. Han H, Segal AM, Seifter JL, et al. Nutritional Management of Kidney Stones (Nephrolithiasis). Clin Nutr Res 2015;4:137-52.

3. Zeng G, Mai Z, Xia S, et al. Prevalence of kidney stones in China: an ultrasonography based cross-sectional study. BJU Int 2017;120:109-16.

4. Parks JH, Coe FL. An increasing number of calcium oxalate stone events worsens treatment outcome. Kidney Int 1994;45:1722-30.

5. Fujii Y, Okada A, Yasui T, et al. Effect of adiponectin on kidney crystal formation in metabolic syndrome model mice via inhibition of inflammation and apoptosis. PLoS One 2013;8:e61343.

6. Khan SR. Reactive Oxygen Species as the Molecular Modulators of Calcium Oxalate Kidney Stone Formation: Evidence from Clinical and Experimental Investigations. J Urol 2013;189:803-11.

7. Lee HJ, Jeong SJ, Park MN, et al. Gallotannin Suppresses
Calcium Oxalate Crystal Binding and Oxalate-Induced Oxidative Stress in Renal Epithelial Cells. Biol Pharm Bull 2012;35:539-44.

8. Antonelli JA, Maalouf NM, Pearle MS, et al. Use of the National Health and Nutrition Examination Survey to calculate the impact of obesity and diabetes on cost and prevalence of urolithiasis in 2030. Eur Urol 2014;66:724-9.

9. Evan AP. Physiopathology and etiology of stone formation in the kidney and the urinary tract. Pediatr Nephrol 2010;25:831-41.

10. Khan SR, Pearle MS, Robertson WG, et al. Kidney stones. Nat Rev Dis Primers 2016;2:16008.

11. de Water R, Leenen PJ, Noordermeer C, et al. Cytokine production induced by binding and processing of calcium oxalate crystals in cultured macrophages. Am J Kidney Dis 2001;38:331-8.

12. Evan AP, Lingeman JE, Worcester EM, et al. Renal histopathology and crystal deposits in patients with small bowel resection and calcium oxalate stone disease. Kidney Int 2010;78:310-7.

13. Moutzouri E, Tsimihodimos V, Tselepis AD. Inflammatory biomarkers and cardiovascular risk assessment. Current knowledge and future perspectives. Curr Pharm Des 2013;19:3827-40.

14. Pineda C, Fuentes-Gomez AJ, Hernandez-Diaz C, et al. Animal model of acute gout reproduces the inflammatory and ultrasonographic joint changes of human gout. Arthritis Res Ther 2015;17:37.

15. Nardi J, Nascimento S, Goethel G, et al. Inflammatory and oxidative stress parameters as potential early biomarkers for silicosis. Clin Chim Acta 2018;484:305-13.

16. Canales BK, Anderson L, Higgins L, et al. Second prize: Comprehensive proteomic analysis of human calcium oxalate monohydrate kidney stone matrix. J Endourol 2008;22:1161-7.

17. Ohtani H. Focus on TILs: prognostic significance of tumor infiltrating lymphocytes in human colorectal cancer. Cancer Immun 2007;7:4.

18. Capone M, Giannarelli D, Mallardo D, et al. Baseline neutrophil-to-lymphocyte ratio (NLR) and derived NLR could predict overall survival in patients with advanced melanoma treated with nivolumab. J Immunother Cancer 2018;6:74.

19. Lech M, Rommele C, Anders HJNDT. Pentraxins in nephrology: C-reactive protein, serum amyloid $\mathrm{P}$ and 
pentraxin-3. 2013;28:803-11.

20. Ansar W, Ghosh SJIR. C-reactive protein and the biology of disease. Immunol Res 2013;56:131-42.

Cite this article as: Mao $\mathrm{W}, \mathrm{Wu} \mathrm{J}$, Zhang $\mathrm{Z}, \mathrm{Xu} \mathrm{Z}, \mathrm{Xu} \mathrm{B}$, Chen M. Neutrophil-lymphocyte ratio acts as a novel diagnostic biomarker for kidney stone prevalence and number of stones passed. Transl Androl Urol 2021;10(1):77-86. doi: 10.21037/tau20-890
21. Shoag J, Eisner BH. Relationship between C-reactive protein and kidney stone prevalence. J Urol 2014;191:372-5. 